\title{
Otimização do processo de extrusão termoplástica da mistura castanha do Brasil com farinha de mandioca
}

\author{
Optimization of the process of thermoplastic extrusion of mixtures of Brazil nut with cassava flour
}

\author{
Maria Luzenira de SOUZA ${ }^{1 *}$, Hilary Castle de MENEZES ${ }^{2}$
}

\begin{abstract}
Resumo
O objetivo deste trabalho foi otimizar as condições de processamento por extrusão termoplástica de misturas de torta de castanha do Brasil com farinha de mandioca, em função da aceitabilidade. Foi feito o delineamento fatorial completo $2^{3}$ para a produção dos extrudados e a avaliação dos ensaios extrusados em função das respostas de aceitação. No estudo de aceitação global aplicou-se o teste afetivo de escala hedônica com 9 pontos e no de intenção de compra o de 5 pontos. O resultado da metodologia de superfície de resposta indica que aumentos de castanha do Brasil, temperatura e umidade elevam as notas de aceitação global e intenção de compra do produto, com uma região de otimização do processamento nas seguintes condições: temperatura entre 120 a $160{ }^{\circ} \mathrm{C}$, castanha entre 48 a $80 \%$ e umidade entre 20 a $25 \%$. $\mathrm{Na}$ torta de castanha, a temperatura e a umidade da mistura muito alta ou muito baixa diminuem as notas de aceitação global e a intenção de compra. As maiores notas de aceitação global e a intenção de compra neste experimento estão nos pontos centrais e indicam a validade do modelo.
\end{abstract}

Palavras-chave: extrusão termoplástica; castanha do Brasil; metodologia de superfície de resposta; aceitação.

\begin{abstract}
The objective of this work was to optimize the conditions of processing mixtures of Brazil nut with cassava flour through thermoplastic extrusion as a function of acceptability. A $2^{3}$ full factorial design was used for the production of the extrudates and the evaluation of the products was according to acceptability responses. An affective hedonic scale of 9 points was applied to evaluate global acceptability and a 5-point scale was used to evaluate purchase intention. The results of the response surface methodology indicated that increases in Brazil nut percentage, temperature and moisture increased global acceptability and purchase intention of the product, with an optimized processing region in the following conditions: temperature between 120 and $160{ }^{\circ} \mathrm{C}$, Brazil nut percentage between 48 and $80 \%$ and moisture between 20 and $25 \%$. If Brazil nut percentage, temperature and moisture of the mixture were too high or too low, global acceptability and purchase intention decreased. The highest acceptance and purchase intention in this study were found for the central points and indicate the validity of the model.
\end{abstract}

Keywords: thermoplastic extrusion; Brazil nut; surface response methodology; acceptance.

\section{Introdução}

O processamento de alimentos por extrusão termoplástica tem destaque e expansão na indústria de alimentos, amplia a variedade de produtos alimentícios disponíveis no mercado e apresenta vantagens que os sistemas convencionais de processamento não oferecem (ESTEVEZ; ESCOBAR; VASQUEZ, 1995). A técnica de extrusão possibilita a obtenção de um efeito nutricional vantajoso e útil no alimento, uma vez que viabiliza a utilização de varias misturas de diferentes matérias-primas ricas em proteínas e amidos, podendo incorporar na composição, minerais e vitaminas, além de possibilitar uma longa vida de prateleira e baixo custo ao produto. É um mecanismo efetivo na preservação, aumenta a vida útil de alimentos, uma vez que destrói microrganismos e inativa complexos enzimáticos e componentes antinutricionais (ANDERSON; HEDLUND, 1990; GUZMAN; LEE; CHICHESTER, 1992; SILVA, 1990).
Variações nas condições de operação de extrusão permitem a produção de extrudados com várias características e que podem ser aplicados na indústria de alimentos e outras (EL-DASH, 1982; 1984).

Durante o processamento de extrusão, as condições especiais existentes dentro do extrusor, tais como: baixo teor de umidade, alta taxa de cisalhamento e alta temperatura, provocam uma série de modificações nas propriedades físico-químicas do produto. Dois mecanismos são considerados responsáveis por estas modificações: a gelatinização e a dextrinização do amido (GROSSMANN, 1986).

A castanha do Brasil (Bertholletia excelsa, H.B.K.) rica em proteína, a única completa entre os alimentos vegetais, e a mandioca (Manihot esculenta, Crantz) fonte de carboidratos, são

Recebido para publicação em 22/6/2007

Aceito para publicação em 25/2/2008 (002507)

${ }^{1}$ Centro de Ciências Biológicas e da Natureza, Unidade de Tecnologia de Alimentos, Universidade Federal do Acre - UFAC, Campus Universitário BR 364, Km 4, CEP 69915-900, Rio Branco - AC, E-mail: mluzen@hotmail.com

${ }^{2}$ Departamento de Tecnologia de Alimentos, Faculdade de Engenharia de Alimentos - FEA, Universidade Estadual de Campinas - UNICAMP, CP 6121, CEP 13083-970, Campinas - SP, E-mail: hilary@mpc.com.br

${ }^{*}$ A quem a correspondência deve ser enviada 
matérias-primas importantes para a subsistência e economia da população Amazônica (PACHECO; SCUSSEL, 2006; SOUZA, 2003; SOUZA; MENEZES, 2004).

Neste contexto, considerando o elevado valor nutricional da castanha do Brasil, especialmente a alta qualidade biológica da proteína, a presença de vários minerais, inclusive o oligoelemento selênio (SOUZA, 2003), a sua baixa utilização pelas indústrias de alimentos brasileiras, a elevada aplicação no mercado internacional, a grande produção de mandioca e a praticidade do processo de extrusão termoplástica, realizou-se este trabalho com o objetivo de otimizar as condições de processamento por extrusão termoplástica de misturas de castanha do Brasil com farinha de mandioca, em função da aceitabilidade de consumidores.

\section{Material e métodos}

\subsection{Material}

Foram utilizados produtos extrudados formulados a partir da mistura de torta de amêndoa de castanha do Brasil semidesengordurada com farinha de mandioca (SOUZA; MENEZES, 2004).

\subsection{Delineamento experimental}

Para a obtenção dos extrudados da mistura foi usado o delineamento fatorial completo $2^{3}$ composto (BARROS NETO; SCARMINIO; BRUNS, 1995; BARROS NETO; SCARMINIO; BRUNS, 2001), contendo as variáveis independentes $\mathrm{X}_{1}=\%$ torta de castanha; $\mathrm{X}_{2}=\%$ umidade; $\mathrm{X}_{3}=$ temperatura ${ }^{\circ} \mathrm{C} \mathrm{em}$ 2 níveis codificados: $-\alpha,-1,0,+1,+\alpha$, conforme apresentado na Tabela 1. O valor de $\alpha$ foi em função do número de variáveis independentes $(n=3)$, sendo definido pela Equação 1:

$\alpha=\left(2^{n}\right)^{1 / 4}=\left(2^{3}\right)^{1 / 4}=1,68$

As faixas de variação entre os limites inferior e superior de cada variável independente foram estabelecidas a partir de dados da literatura e de testes preliminares realizados.

O planejamento experimental foi constituído por 18 ensaios (Tabela 2), sendo cada ensaio constituído por torta de castanha e farinha de mandioca, determinando-se os percentuais de farinha de mandioca de cada um, através da diferença entre 100 e a porcentagem de torta de castanha e a porcentagem de umidade, através da fórmula: volume de água $=\left(\mathrm{U}_{\mathrm{f}}-\mathrm{U}_{\mathrm{i}}\right) /\left(100-\mathrm{U}_{\mathrm{f}}\right) \times$ pa.

Tabela 1. Variáveis independentes codificadas e reais com seus diferentes níveis.

\begin{tabular}{crrrrr}
\hline Variáveis & \multicolumn{5}{c}{ Níveis codificados e reais } \\
\cline { 2 - 6 } independentes & \multicolumn{1}{c}{$\alpha$} & -1 & 0 & +1 & $+\alpha$ \\
\hline $\mathrm{X}_{1}$ & 19,80 & 30 & 45 & 60 & 70,2 \\
$\mathrm{X}_{2}$ & 14,20 & 17 & 21 & 25 & 27,7 \\
$\mathrm{X}_{3}$ & 116,40 & 130 & 150 & 170 & 183,6 \\
\hline
\end{tabular}

$\mathrm{X}_{1}$ (torta de castanha \%); $\mathrm{X}_{2}($ umidade $\%) ; \mathrm{X}_{3}\left(\right.$ temperatura $\left.{ }^{\circ} \mathrm{C}\right)$; níveis codificados: $\pm \alpha ; 0 ; \pm 1$; pontos axiais ou estrela $\pm \alpha= \pm 1,68$; ponto central $C=0$; pontos máximos e mínimos $= \pm 1$; e níveis reais: valores de 14,2 a 183,6 .
Em que: $\mathrm{U}_{\mathrm{f}}=$ umidade final; $\mathrm{U}_{\mathrm{i}}=$ umidade inicial; $\mathrm{pa}=$ peso em g da amostra.

Os ensaios foram preparados, homogeneizados, embalados em sacos de polietileno, selados e armazenados sob refrigeração a $5^{\circ} \mathrm{C}$ por 24 horas para uniformizar a umidade e antes da extrusão.

A extrusão foi processada em extrusor marca Brabender de rosca única, com as seguintes características: $380 \mathrm{~mm}$ de comprimento por $19 \mathrm{~mm}$ de diâmetro, taxa de compressão 3:1 com matriz cilíndrica de $2 \mathrm{~mm}$ de diâmetro, pertencente ao Laboratório de Cereais da Faculdade de Engenharia de Alimentos da Universidade Estadual de Campinas. O processamento teve início quando as 3 diferentes zonas do extrusor atingiram as temperaturas pré-estabelecidas, mantendo-se constantes as temperaturas da $1^{\mathrm{a}}$ zona $=100^{\circ} \mathrm{C}$ e da $2^{\mathrm{a}}$ zona $=110^{\circ} \mathrm{C}$; enquanto as temperaturas utilizadas na $3^{\mathrm{a}}$ zona foram aquelas definidas para cada ensaio (tratamento) determinadas no planejamento (Tabela 2). E a taxa de alimentação da mistura foi mantida constante a 4,8 kg/h com $175 \mathrm{rpm}$ (SOUZA, 2003).

Depois que o processo atingiu o equilíbrio na leitura visual do torque do extrusor, o material extrudado foi coletado manualmente, cortado em pedaços de cerca de $3 \mathrm{~cm}$, distribuído em bandejas, desidratado em estufa com circulação de ar a $60^{\circ} \mathrm{C}$ por 48 horas, esfriado em temperatura ambiente, pesado, embalado em saco flexível aluminizado multicamada e selado, e armazenado em local seco e fresco no laboratório do Departamento de Tecnologia de Alimentos da Unicamp até a realização das análises sensoriais (SOUZA, 2003).

Tabela 2. Planejamento fatorial completo composto central $\left(2^{3}\right)$, com 3 variáveis independentes, 4 repetições no ponto central $(C)$ e 6 pontos axiais da mistura torta de castanha do Brasil com farinha de mandioca.

\begin{tabular}{cccc}
\hline $\begin{array}{c}\text { No ensaio } \\
\text { (Tratamento) }\end{array}$ & \multicolumn{3}{c}{ Níveis das variáveis independentes codificadas e reais } \\
\cline { 2 - 4 } & $\begin{array}{c}\mathrm{X}_{1}(\%) \text { Torta de } \\
\text { castanha }\end{array}$ & $\begin{array}{c}\mathrm{X}_{2}(\%) \\
\text { Umidade }\end{array}$ & $\begin{array}{c}\mathrm{X}_{3}\left({ }^{\circ} \mathrm{C}\right) \\
\text { Temperatura }\end{array}$ \\
\hline 01 & $-1(30)$ & $-1(17)$ & $-1(130)$ \\
02 & $+1(60)$ & $-1(17)$ & $-1(130)$ \\
03 & $-1(30)$ & $+1(25)$ & $-1(130)$ \\
04 & $+1(60)$ & $+1(25)$ & $-1(130)$ \\
05 & $-1(30)$ & $-1(17)$ & $+1(170)$ \\
06 & $+1(60)$ & $-1(17)$ & $+1(170)$ \\
07 & $-1(30)$ & $+1(25)$ & $+1(170)$ \\
08 & $+1(60)$ & $+1(25)$ & $+1(170)$ \\
09 & $0(45)$ & $0(21)$ & $-\alpha 1,68(116,4)$ \\
10 & $0(45)$ & $0(21)$ & $+\alpha 1,68(183,6)$ \\
11 & $0(45)$ & $-\alpha 1,68(14,28)$ & $0(150)$ \\
12 & $0(45)$ & $+\alpha 1,68(27,72)$ & $0(150)$ \\
13 & $-\alpha 1,68(19,8)$ & $0(21)$ & $0(150)$ \\
14 & $+\alpha 1,68(70,2)$ & $0(21)$ & $0(150)$ \\
$15(\mathrm{C})$ & $0(45)$ & $0(21)$ & $0(150)$ \\
$16(\mathrm{C})$ & $0(45)$ & $0(21)$ & $0(150)$ \\
17 (C) & $0(45)$ & $0(21)$ & $0(150)$ \\
$18(\mathrm{C})$ & $0(45)$ & $0(21)$ & $0(150)$ \\
\hline
\end{tabular}

$\mathrm{X}_{1}=$ torta de castanha $(\%) ; \mathrm{X}_{2}=$ umidade $(\%) ; \mathrm{X}_{3}=$ temperatura $\left({ }^{\circ} \mathrm{C}\right) ; \mathrm{C}=$ ponto central Valores codificados: $\pm \alpha ; 0 \mathrm{e} \pm 1$ representam $\pm \alpha$ (pontos axiais ou estrela $= \pm 1,68$ ); 0 (zero) (ponto central C) e \pm 1 (pontos máximos e mínimos das variáveis independentes). 


\subsection{Análise sensorial}

\section{Teste de aceitação}

Foi realizado o teste afetivo de aceitação usando a escala hedônica de 9 pontos para avaliar a aceitação global (PERYAM; GIRARDOT, 1952) e de 5 pontos para intenção de compra. As amostras foram codificadas em números aleatórios de 3 dígitos, foram apresentadas ao acaso em 6 sessões, em cabines individuais sob iluminação ambiente com luz vermelha (MEILGAARD; CIVILLE; CARR, 1987; STONE; SIDEL, 1985) a 42 provadores não treinados, de ambos os sexos, pertencentes à academia da Universidade Estadual de Campinas, que avaliaram amostras dos 18 ensaios do produto, em 6 diferentes sessões, assinalando em uma ficha de avaliação (Quadro 1) as suas notas para aceitação global e intenção de compra.

\subsection{Análise estatística}

Os resultados das variáveis dependentes, respostas de cada um dos 18 ensaios, foram tratados por análise de regressão múltipla da metodologia de superfície de resposta, e a análise de variância (ANOVA) foi aplicada para testar a adequação dos modelos. Nos modelos foi observada a significância da regressão

Quadro 1. Ficha modelo para avaliação sensorial dos ensaios extrusados.

Provador no__ Nome:
E-mail:
Fone:
1. Você está recebendo 3 amostras codificadas de um produto ex-
trudado formulado com torta de amêndoa de castanha do Brasil e
farinha de mandioca. Por favor, prove-os avaliando a aceitação glo-
bal, utilizando as notas de 1 a 9 em relação a quanto você gostou ou
desgostou:
9 - Adorei
8 - Gostei muito
7 - Gostei moderadamente
6 - Gostei ligeiramente
5 - Nem gostei/nem desgostei
4 - Desgostei ligeiramente
3 - Desgostei moderadamente
2 - Desgostei muito
1 - Detestei Comentário
Amostra Nota C Co

$\overline{\text { 2. Com base em sua opinião sobre as } 3 \text { amostras, indique na escala }}$ de 1 a 5 sua nota em relação a sua atitude, caso você encontrasse cada uma das amostras à venda. Se eu encontrasse este produto à venda eu:

5 - Certamente compraria

4 - Possivelmente compraria

3 - Talvez comprasse/talvez não comprasse

2 - Possivelmente não compraria

1 - Certamente não compraria

Amostra Nota Comentário pelo teste $F$ e pelo coeficiente de determinação (BARROS NETO; SCARMINIO; BRUNS, 1995; BARROS NETO; SCARMINIO; BRUNS, 2001; BOX; DRAPER, 1987). Para visualizar os efeitos das variáveis independentes e de suas interações sobre as respostas avaliadas, a aceitação global e a intenção de compra, conforme o planejamento fatorial completo (Tabela 4), foi construído um gráfico de superfície de respostas quando a ANOVA mostrou-se significativa.

Determinou-se o ajuste do modelo de segunda ordem e a otimização das melhores condições de extrusão (BARROS NETO; SCARMINIO; BRUNS, 1995; BARROS NETO; SCARMINIO; BRUNS, 2001; BOX; DRAPER, 1987), considerando-se as melhores condições das variáveis independentes e suas interações sobre as respostas, maximizando a resposta desejada e/ou minimizando a indesejada. O processamento dos dados foi realizado com o programa Statistic for Windows versão 5.0 (1995).

\section{Resultados e discussão}

\subsection{Efeitos das variáveis de extrusão sobre a aceitabilidade dos produtos extrudados}

$\mathrm{Na}$ Tabela 3 estão apresentadas as médias dos resultados do planejamento experimental obtidas dos testes de avaliação da aceitação global e intenção de compra dos produtos extrudados.

A partir dos resultados experimentais das variáveis estudadas (Tabela 3), estabeleceram-se os coeficientes de regressão a 5\% de significância. Os resultados dos fatores Lineares (L) e quadráticos $(\mathrm{Q})$ e das interações para aceitação global e intenção de compra dos ensaios extrusados encontram-se na Tabela 4. Em relação à aceitação global, todos os parâmetros lineares e quadráticos das três variáveis, bem como suas interações foram significativos $(\mathrm{p} \leq 0,05)$.

Os parâmetros Lineares (L) das variáveis independentes, castanha e umidade, apresentaram efeitos positivos sobre a aceitação global, indicando que um aumento em qualquer um destes fatores contribui para a elevação na aceitação global do extrudado, o mesmo ocorrendo com as interações lineares entre castanha $\mathrm{X}$ umidade e umidade $\mathrm{X}$ temperatura.

A temperatura linear e a interação castanha $x$ temperatura linear negativas mostram que a elevação destes fatores indica um efeito negativo, ou seja, uma diminuição de notas na resposta do teste aceitação global. O parâmetro castanha (L) foi o de maior valor absoluto, exercendo um efeito positivo de 1,3 vezes superior aos demais, em relação à aceitação global.

Os parâmetros castanha, umidade e temperatura (quadráticos) apresentaram efeitos com valores absolutos negativos, indicando a existência de uma região de máxima e a superfície de resposta, na forma de uma parábola negativa, isto é, com curvatura voltada para baixo.

A análise de variância (ANOVA) obtida para aceitação global está apresentada na Tabela 5 . O modelo de regressão gerado foi significativo $(\mathrm{p} \leq 0,05)$ porque o $\mathrm{F}_{\text {calculado }}(0,95 ; 9 ; 8)=9,52$ foi maior que o $\mathrm{F}_{\text {tabelado }}(0,95 ; 9: 8)=3,39$. Porém, a falta de ajuste também foi significativa $\left(\mathrm{F}_{\text {calculado }}(0,95 ; 5 ; 3)=364,65>\mathrm{F}_{\text {tabelado }}\right.$ 
Tabela 3. Planejamento fatorial completo $2^{3}$ e a média dos resultados das respostas dos testes de aceitação.

\begin{tabular}{|c|c|c|c|c|c|}
\hline \multirow[t]{2}{*}{ No ensaio (tratamento) } & \multicolumn{3}{|c|}{ Níveis das variáveis independentes codificados e reais } & \multicolumn{2}{|c|}{ Respostas sensoriais } \\
\hline & $\mathrm{X}_{1}$ Torta de castanha $(\%)$ & $\mathrm{X}_{2}$ Umidade $(\%)$ & $\mathrm{X}_{3}$ Temperatura $\left({ }^{\circ} \mathrm{C}\right)$ & Aceitação global & Intenção de compra \\
\hline 01 & $-1(30)$ & $-1(17)$ & $-1(130)$ & 5,57 & 3,02 \\
\hline 02 & $+1(60)$ & $-1(17)$ & $-1(130)$ & 7,12 & 3,50 \\
\hline 03 & $-1(30)$ & $+1(25)$ & $-1(130)$ & 5,67 & 3,04 \\
\hline 04 & $+1(60)$ & $+1(25)$ & $-1(130)$ & 7,17 & 3,66 \\
\hline 05 & $-1(30)$ & $-1(17)$ & $+1(170)$ & 4,57 & 2,45 \\
\hline 08 & $+1(60)$ & $+1(25)$ & $+1(170)$ & 7,10 & 3,50 \\
\hline 09 & $0(45)$ & $0(21)$ & $-\alpha(116,4)$ & 6,05 & 3,16 \\
\hline 10 & $0(45)$ & $0(21)$ & $+\alpha(183,6)$ & 5,36 & 2,88 \\
\hline 11 & $0(45)$ & $-\alpha(14,28)$ & $0(150)$ & 3,52 & 1,90 \\
\hline 12 & $0(45)$ & $+\alpha(27,72)$ & $0(150)$ & 6,40 & 3,33 \\
\hline $16(C)$ & $0(45)$ & $0(21)$ & $0(150)$ & 7,19 & 3,90 \\
\hline $17(\mathrm{C})$ & $0(45)$ & $0(21)$ & $0(150)$ & 7,12 & 3,64 \\
\hline $18(\mathrm{C})$ & $0(45)$ & $0(21)$ & $0(150)$ & 7,17 & 3,73 \\
\hline
\end{tabular}

$\mathrm{X}_{1}=$ torta de castanha $(\%) ; \mathrm{X}_{2}=$ umidade $(\%) ; \mathrm{X}_{3}=$ temperatura $\left({ }^{\circ} \mathrm{C}\right) ; \mathrm{C}=$ ponto central. Valores codificados: $\pm \alpha ; 0$ e \pm 1 representam $\pm \alpha($ pontos axiais ou estrela $= \pm 1,68) ; 0($ zero $)($ ponto central C); e \pm 1 (pontos máximos e mínimos das variáveis independentes).

Tabela 4. Estimativa do coeficiente de regressão e teste $T$ sobre as respostas de aceitação global e intenção de compra dos extrusados.

\begin{tabular}{|c|c|c|c|c|c|c|c|}
\hline \multirow[t]{3}{*}{ Variáveis independentes } & \multirow{3}{*}{$\begin{array}{l}\text { Parâmetros do } \\
\text { modelo }\end{array}$} & \multicolumn{6}{|c|}{ Coeficientes estimados } \\
\hline & & \multicolumn{3}{|c|}{ Aceitação global } & \multicolumn{3}{|c|}{ Intenção de compra } \\
\hline & & Coeficiente & teste $T$ & Erro puro & Coeficiente & teste $T$ & Erro puro \\
\hline Média & $\beta_{\widetilde{0}}$ & $7,13^{*}$ & $459,0^{*}$ & $0,015^{\star}$ & $3,77^{\star}$ & $64,3^{*}$ & $0,058^{*}$ \\
\hline (1) Castanha (L) & $\beta_{1}$ & $1,30^{*}$ & $77,4^{*}$ & $0,01^{\star}$ & $0,55^{\star}$ & $8,78^{*}$ & $0,06^{*}$ \\
\hline Castanha (Q) & $\beta_{11}$ & $-0,39^{*}$ & $-22,3^{*}$ & $0,01^{\star}$ & $-0,27^{\star}$ & $-4,12^{*}$ & $0,06^{\star}$ \\
\hline (2) Umidade (L) & $\beta_{2}$ & $1,09^{*}$ & $64,8^{*}$ & $0,01^{\star}$ & $0,56^{*}$ & $8,87^{\star}$ & $0,06^{*}$ \\
\hline Temperatura (Q) & $\beta_{33}$ & $-0,84^{*}$ & $-48,2^{*}$ & $0,01^{\star}$ & $-0,48^{\star}$ & $-7,33^{*}$ & $0,06^{*}$ \\
\hline $1 \mathrm{~L} \times 2 \mathrm{~L}$ & $\beta_{12}$ & $0,20^{*}$ & $9,43^{*}$ & $0,02^{*}$ & 0,19 & 2,37 & 0,08 \\
\hline $1 \mathrm{~L} \times 3 \mathrm{~L}$ & $\beta_{3}$ & $-0,11^{*}$ & $-5,1^{\star}$ & $0,02^{*}$ & $-0,06$ & 0,812 & 0,08 \\
\hline $2 \mathrm{~L} \times 3 \mathrm{~L}$ & $\beta_{23}$ & $0,57^{*}$ & $26,2^{*}$ & $0,02^{*}$ & $0,27^{\star}$ & $3,28^{*}$ & $0,08^{\star}$ \\
\hline
\end{tabular}

${ }^{*}$ Significativo a $\mathrm{p} \leq 0,05$.

Tabela 5. Análise de variância (ANOVA) do modelo completo da tabela de regressão ( $\mathrm{p} \leq 0,05)$ para o teste de aceitação global.

\begin{tabular}{lccccc}
\hline Fonte de variação & Soma quadrática & Graus de liberdade & Média quadrática & $\mathrm{F}_{\text {Calculado }}(\mathrm{p} \leq 0,05)$ & $\mathrm{F}_{\text {tabelado }}(\mathrm{p} \leq 0,05)$ \\
\hline Regressão & 18,919 & 9 & 2,102 & - & 3,39 \\
Resíduo & 1,765 & 8 & 0,220 & 364,65 & - \\
Falta de ajuste & 1,762 & 5 & 0,352 & - & - \\
Erro puro & 0,002 & 3 & 0,000 & - \\
Total & 20,684 & 17 & 1,216 & - \\
\hline
\end{tabular}

$\mathrm{R}^{2}=$ Coeficiente de determinação 0,9146 .

$(0,95 ; 5 ; 3)=9,01)$, embora o ideal seria um valor de $F_{\text {calculado }}$ menor que o $\mathrm{F}_{\text {tabelado }}$, ou seja, não significativo. Entretanto, como as médias nos pontos centrais foram muito próximas e o erro puro muito baixo (razões para o F da falta de ajuste alto), o modelo foi considerado válido para fins preditivos (BARROS NETO;
SCARMINIO; BRUNS, 1995; BARROS NETO; SCARMINIO; BRUNS, 2001).

De acordo com Waszczynsky e Nelsen (1996), quando o quadrado médio para o erro experimental tomar valor extre- 
mamente baixo, os testes de significância para a falta de ajuste devem ser considerados irrelevantes, o que corrobora com os achados neste trabalho.

O coeficiente de determinação da regressão $\left(R^{2}\right)=0,9146$ para o modelo foi válido, indicando que ele explica $91,46 \%$ da variação dos dados observados. Conforme Barros Neto, Scarminio e Bruns (1995; 2001); Khuri e Cornell (1996), o coeficiente de determinação $\left(\mathrm{R}^{2}\right)$ mede a proporção da variação total da resposta que é explicada pelo modelo. Desse modo, quanto maior o $\mathrm{R}^{2}$, isto é, quanto mais próximo de 1 , menor será o erro e melhor o modelo. Segundo estes autores, modelos com $\mathrm{R}^{2}<0,60$ devem ser usados somente como indicadores de tendência, nunca para fins preditivos.

Pelo teste $T$ aplicado, os coeficientes de regressão significativos a 95\% foram considerados nos modelos matemáticos propostos para representar as Equações 1 (AG - Aceitação Global) e 2 (IC - Intenção de Compra) dos ensaios extrudados, em função da torta de castanha, umidade e temperatura. Segue abaixo a Equação 1:

$\mathrm{AG}=7,13+1,30 \mathrm{TC}-0,39 \mathrm{TC}^{2}+1,09 \mathrm{U}-1,37 \mathrm{U}^{2}-0,61 \mathrm{~T}-$

$0,84 \mathrm{~T}^{2}+0,20 \mathrm{TC} . \mathrm{U}-0,11 \mathrm{TC} . \mathrm{T}+0,57 \mathrm{U} . \mathrm{T}$

As superfícies de respostas e de contornos para a aceitação global dos extrudados, baseadas no modelo gerado pela Equação 1, estão ilustradas nas Figuras 1a, $1 \mathrm{~b}$ e $1 \mathrm{c}$, onde se observa o efeito positivo da castanha na aceitação global do produto, sendo que quanto maior o percentual de castanha maior a aceitação global, com uma região de máxima.

Para a otimização foi fixada a umidade no ponto central (21\%) e variou-se o percentual de castanha $\mathrm{x}$ temperatura, $\mathrm{e}$ verifica-se que a partir de $48 \%$ de castanha foi aumentando o nível de aceitação, com a região otimizada na faixa entre 48 a $80 \%$ de torta de castanha, ao mesmo tempo em que a temperatura de extrusão na região da faixa entre 120 a $160{ }^{\circ} \mathrm{C}$ mostrou-se como a melhor região otimizada, com maior aceitação global para o produto.

Em relação ao teste de intenção de compra, as médias dos resultados obtidos também estão apresentadas na Tabela 3. A análise foi feita da mesma forma da atenção global, considerando-se os coeficientes de regressão a 5\% de significância (Tabela 4).

Os parâmetros lineares das variáveis independentes de castanha e umidade, bem como a interação umidade $X$ temperatura apresentaram efeitos positivos para a intenção de compra, indicando que um aumento em qualquer um destes fatores implica no aumento da intenção de compra do produto.

O parâmetro temperatura linear negativa indica que elevando-se a temperatura se reduz a intenção de compra. Os parâmetros quadráticos torta de amêndoa de castanha, umidade e temperatura apresentaram valores negativos, indicando a existência de uma região de máxima e a superfície de resposta formando uma parábola negativa, ou seja, com curvatura voltada para baixo. Isto explica que existe um limite destes fatores para se atingir um ponto máximo, ou seja, a otimização para a intenção de compra.
Nos efeitos estimados dos coeficientes de regressão dos fatores lineares quadráticos e interações das variáveis independentes para o teste intenção de compra observa-se que dos 10 parâmetros do modelo, 8 foram significativos $(\mathrm{p} \leq 0,05)$ e permaneceram no modelo para construção da análise de variância (ANOVA) ajustada, apresentada na Tabela 6.

A ANOVA (Tabela 6) mostra uma regressão significativa $(\mathrm{p} \leq 0,05)$ e mostra que o resíduo não apresentou evidência de falta de ajuste, pois o $\mathrm{F}_{\text {calculado }}(0,95 ; 7 ; 10)=\mathrm{MQ}_{\mathrm{R}} / \mathrm{MQ}_{\mathrm{r}}=13,62$ maior que o $\mathrm{F}_{\text {tabelado }}(0,95 ; 7 ; 10)=3,14$ comprova uma equação com o $\mathrm{F}_{\text {calculado }} 4,33$ vezes superior ao $\mathrm{F}_{\text {tabelado }}$, anunciando uma equação válida, significativa estatisticamente e útil para fins preditivos, corroborando com Barros Neto, Scarminio e Bruns $(1995 ; 2001)$, que reportam que o valor de $\mathrm{F}_{\text {calculado }}$ deve ser no mínimo 4 a 5 vezes o valor de $\mathrm{F}_{\text {tabelado }}$ para assegurar que a regressão é significativa estatisticamente e o modelo útil para fins preditivos.

O coeficiente de determinação, $R^{2}=0,9050$, encontrado é significativo $(\mathrm{p} \leq 0,05)$ e o modelo é válido, indicando que ele explica $90,50 \%$ da variação dos dados observados. Este coeficiente mede a proporção da variação total da resposta que é explicada pelo modelo (BARROS NETO; SCARMINIO; BRUNS, 1995; BARROS NETO; SCARMINIO; BRUNS, 2001; KHURI; CORNELL, 1996). Desse modo, quanto maior o $\mathrm{R}^{2}$ menor será o erro e melhor o modelo.

Aplicou-se o teste $T$ aos coeficientes de regressão significativos a $(\mathrm{p} \leq 0,05)$, considerando-se o modelo matemático para representar a Equação 2 (IC = intenção de compra) da resposta dos ensaios extrudados, em função das variáveis independentes, torta de castanha, umidade e temperatura, representada por um polinômio de segunda ordem, conforme demonstrado:

$\mathrm{IC}=3,77+0,55 \mathrm{TC}-0,27 \mathrm{TC}^{2}+0,56 \mathrm{U}-$

$0,77 \mathrm{U}^{2}-0,35 \mathrm{CT}-0,48 \mathrm{CT}^{2}+0,27 \mathrm{U} . \mathrm{CT}$

Com base neste modelo, foram construídas superfícies de respostas e de contornos para a intenção de compra, conforme Figuras 2a, b e c. Observa-se nas figuras citadas, que ao se fixar a umidade no ponto central e variando a castanha $X$ temperatura, verificou-se a elevação nas notas de intenção de compra a partir de 48 a $80 \%$ de castanha, com 20 a $25 \%$ de umidade e 120 a $160^{\circ} \mathrm{C}$ de temperatura de extrusão, sendo estas as faixas em que se obtêm a região otimizada para intenção de compra.

Por outro lado, quando a temperatura de extrusão e umidade da mistura está muito alta e/ou muito baixa, reduzemse as notas de aceitação global e a intenção de compra dos extrusados.

No planejamento experimental substituíram-se as variáveis independentes pelos valores codificados, obtendo-se os valores preditivos e os desvios padrão (Tabela 7). Analisando-se os resultados das equações de modelo predito e os resultados experimentais, para as respostas de aceitação global e de intenção de compra, encontram-se respostas nos pontos centrais muito próximos e com baixo desvio padrão, o que indica a validade do modelo. Desse modo, o processamento por extrusão termoplástica da mistura pode ser otimizado, fixando-se as variáveis independentes em um ou mais destes pontos centrais em que 

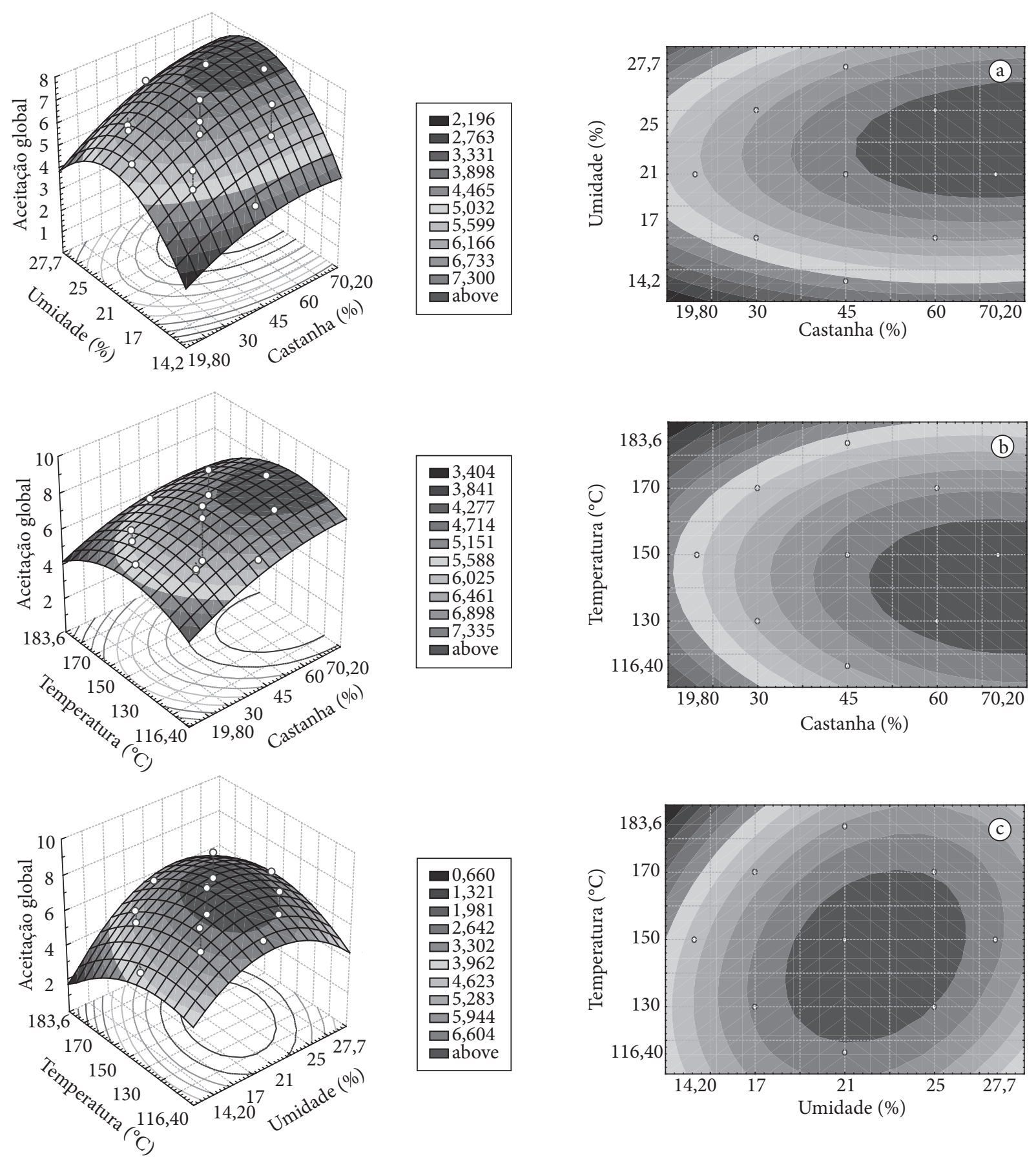

Figura 1. a) Superfície de resposta e contorno mostrando o efeito das variáveis de extrusão sobre a aceitação global, para a temperatura fixa no ponto central, nível zero $\left(150^{\circ} \mathrm{C}\right)$; b) Superfície de resposta e contorno mostrando o efeito das variáveis de extrusão sobre a aceitação global, para a umidade fixa no ponto central, nível zero (21\%); e c) Superfície de resposta e contorno mostrando o efeito das variáveis de extrusão sobre a aceitação global, para a castanha fixa no ponto central, nível zero (45\%).

Tabela 6. Análise de variância (ANOVA) do modelo ajustado $(\mathrm{p} \leq 0,05)$ obtida a partir do coeficiente de regressão das respostas do teste intenção de compra.

\begin{tabular}{lccccc}
\hline Fonte de variação & Soma quadrática & Graus de liberdade & Média quadrática & $\mathrm{F}_{\text {calculado }}(\mathrm{p} \leq 0,05)$ & $\mathrm{F}_{\text {tabelado }}(\mathrm{p} \leq 0,05)$ \\
\hline Regressão & 4,973 & 7 & 0,710 & - & 3,14 \\
Resíduo & 0,521 & 10 & 0,052 & - \\
Falta de ajuste & 0,480 & 7 & 0,068 & - \\
Erro puro & 0,041 & 3 & 0,013 & - \\
Total & 5,494 & 17 & 0,323 & - \\
\hline
\end{tabular}



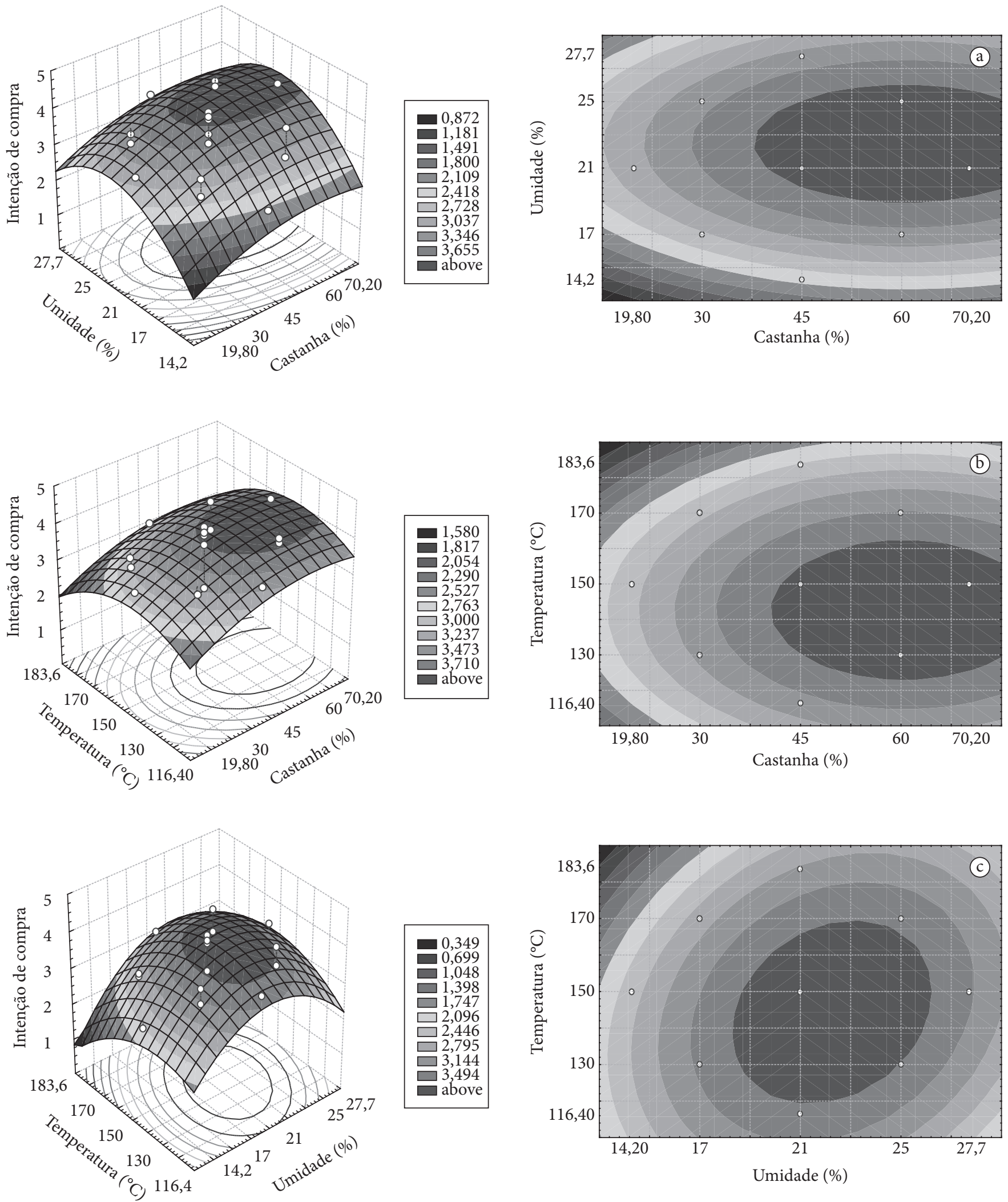

Figura 2. a) Superfície de resposta e contorno mostrando o efeito das variáveis de extrusão sobre a intenção de compra, para a temperatura fixa no ponto central, nível zero $\left(150^{\circ} \mathrm{C}\right)$; b) Superfície de resposta e contorno mostrando o efeito das variáveis de extrusão sobre a intenção de compra, para a umidade fixa no ponto central, nível zero (21\%); e c) Superfície de resposta e contorno mostrando o efeito das variáveis de extrusão sobre a intenção de compra, para a castanha fixa no ponto central, nível zero (45\%). 
Tabela 7. Respostas do delineamento experimental fatorial completo $2^{3}$ referente aos testes de aceitação global e intenção de compra observadas no laboratório e previstas pelo modelo, bem e,como seus respectivos desvio padrão.

\begin{tabular}{|c|c|c|c|c|c|c|c|c|c|}
\hline \multirow{3}{*}{$\begin{array}{c}\text { Ensaio } \\
\text { extrudado }\end{array}$} & \multicolumn{3}{|c|}{ Variáveis independentes (valores codificados) } & \multicolumn{6}{|c|}{ Variáveis dependentes respostas } \\
\hline & \multirow{2}{*}{$\frac{\mathrm{X}_{1}}{\mathrm{C}(\%)}$} & \multirow{2}{*}{$\frac{\mathrm{X}_{2}}{\mathrm{U}(\%)}$} & \multirow{2}{*}{$\frac{\mathrm{X}_{3}}{\mathrm{~T}\left({ }^{\circ} \mathrm{C}\right)}$} & \multicolumn{3}{|c|}{ Aceitação global } & \multicolumn{3}{|c|}{ Intenção de compra } \\
\hline & & & & Obser. & Mod. pre & Desvio padrão & Obser. & Mod. pre & Desvio padrão \\
\hline 01 & -1 & -1 & -1 & 5,57 & 5,28 & 5,21 & 3,02 & 2,76 & 8,51 \\
\hline 02 & 1 & -1 & -1 & 7,12 & 6,49 & 8,88 & 3,5 & 3,32 & 5,09 \\
\hline 03 & -1 & 1 & -1 & 5,67 & 5,59 & 1,47 & 3,04 & 3,06 & $-0,49$ \\
\hline 04 & 1 & 1 & -1 & 7,17 & 7,21 & $-0,55$ & 3,66 & 3,61 & 1,26 \\
\hline 07 & -1 & 1 & 1 & 5,36 & 5,66 & $-5,52$ & 2,76 & 2,97 & $-7,60$ \\
\hline 08 & 1 & 1 & 1 & 7,10 & 7,05 & 0,63 & 3,5 & 3,53 & $-0,81$ \\
\hline 09 & 0 & 0 & $-1,68$ & 6,05 & 6,46 & $-6,84$ & 3,16 & 3,39 & $-7,28$ \\
\hline 10 & 0 & 0 & 1,68 & 5,36 & 5,42 & $-1,16$ & 2,88 & 2,79 & 3,14 \\
\hline 11 & 0 & $-1,68$ & 0 & 3,52 & 4,28 & $-21,62$ & 1,9 & 2,21 & $-16,34$ \\
\hline 15 & 0 & 0 & 0 & 7,14 & 7,13 & 0,09 & 3,85 & 3,77 & 1,97 \\
\hline 16 & 0 & 0 & 0 & 7,19 & 7,13 & 0,78 & 3,9 & 3,77 & 3,23 \\
\hline 17 & 0 & 0 & 0 & 7,12 & 7,13 & $-0,19$ & 3,64 & 3,77 & $-3,67$ \\
\hline 18 & 0 & 0 & 0 & 7,17 & 7,13 & 0,50 & 3,73 & 3,77 & $-1,17$ \\
\hline
\end{tabular}

$\mathrm{X}_{1}=$ torta de castanha (\%); $\mathrm{X}_{2}=$ umidade $(\%) ; \mathrm{X}_{3}=$ temperatura $\left({ }^{\circ} \mathrm{C}\right) ;$ Obser. $=$ resultados das respostas observadas no laboratório; Mod. pre $=$ resultados do modelo preditivo; e $\mathrm{DP}=$ desvio padrão dos resultados.

houve as melhores respostas, ou seja, na região da faixa de otimização.

\section{Conclusões}

Tortas de castanha em temperatura e umidade da mistura muito alta e/ou muito baixa diminuem as notas de aceitação global e intenção de compra dos extrudados, sinalizando uma região ótima.

O processamento por extrusão termoplástica da mistura pode ser otimizado fixando-se as variáveis independentes em um ou mais pontos centrais de melhor resposta.

\section{Agradecimentos}

Os autores agradecem à Universidade Federal do Acre-UFAC, à Universidade Estadual de Campinas - UNICAMP, à Fundação de Amparo à Pesquisa do Estado de São Paulo - FAPESP e ao Conselho Nacional de Desenvolvimento Científico e Tecnológico - CNPq, pela oportunidade, pelo auxílio financeiro ao projeto e pela bolsa de doutorado concedida.

\section{Referências bibliográficas}

ANDERSSON, Y.; HEDLUND, B. Extruded wheat flour: correlation between processing and product quality parameters. Food quality and preference, v. 2, n. 4, p. 201, 1990.

BARROS NETO, B.; SCARMINIO, I. S.; BRUNS, R. E. Planejamento e otimização de experimentos. 2 ed. Campinas: Ed. UNICAMP, 1995. 299 p.
BARROS NETO, B.; SCARMINIO, I. S.; BRUNS, R. E. Como fazer experimentos: pesquisa e desenvolvimento na ciência e na industria. Campinas: EDUNICAMP, 2001.

BOX, G. E. P.; DRAPER, N. R. Empirical model-building and response surfaces. New York: J. Willey \& Sons, 1987. 669 p.

CARVALHO, R. V. Formulações de snacks de terceira geração por extrusão: caracterização texturométrica e microestrutural. Lavras, 2000. 89 f. Dissertação - (Mestrado em Ciência de Alimentos), Universidade Federal de Lavras - UFLA.

EL-DASH, A. A. Termoplastic extrusion of food, theory and techniques. Campinas: UNICAMP, 1982. 81p.

Response surface methodology in the control of thermoplastic extrusion of starch. In: Ronald Jowett (ed.). Extrusion cooking technology. London: Elsevier, 1984. p. 51-74.

ESTEVEZ, A. M.; ESCOBAR, B.; VASQUEZ, E. Cereal and nut bars, nutritional quality and storage stability. Plant food for human nutrition, v. 47, n. 4, p. 309-317, 1995.

GROSSMANN, M. V. E. Efeito da extrusão termoplástica na gelatinização do amido de mandioca visando a produção de álcool. Campinas, 1986. 124 f. Tese - (Doutorado em Tecnologia de Alimentos), Faculdade de Engenharia de Alimentos, Universidade Estadual de Campinas - UNICAMP.

GUZMAN, L. B.; LEE, T. C.; CHICHESTER, C. O. Lipid binding during extrusion cooking. In: KOKINI, J. L..; HO, C. T.; MUKUND, V. K. (eds). Food extrusion science and technology. New York: Marcel Dekker Inc., 1992. cap. 7, p. 427- 436.

KHURI, A. I.; CORNELL, J. A. Response surface: design and analyses. 2 ed. New York: Marcel Dekker Inc., 1996.

MEILGAARD, M.; CIVILLE, G. V.; CARR, B. T. Sensory evaluation techniques. Boca Raton: CRC Press, 1987. v.1, p.113-118. 
PACHECO, A. M.; SCUSSEL, V. M. Castanha-do-Brasil: da floresta tropical ao consumidor. Florianópolis: Editorgraf, 2006. 176 p.

PERYAM, D. R.; GIRARDOT, N. Advanced taste-test method. Food Engineering, v. 24, n. 7, p. 58-61, 1952.

SILVA, M. F. V. Avaliação nutricional de um produto extrusado a base de folha e farinha de mandioca. Lavras, 1990. $100 \mathrm{f}$. Dissertação - (Mestrado em Ciência dos Alimentos), Escola Superior de Agricultura de Lavras - ESAL.

SOUZA,M.L.Processamentodecereaismatinaisextrusadosdecastanhado-brasil com mandioca. Campinas, 2003. $191 \mathrm{f}$. Tese - (Doutorado em Tecnologia de Alimentos), Faculdade de Engenharia de Alimentos, Universidade Estadual de Campinas - UNICAMP.
SOUZA, M. L.; MENEZES, H. C. Processamento de amêndoa e torta de castanha-do-brasil e farinha de mandioca: parâmetros de qualidade. Ciência e Tecnologia de Alimentos, v. 24, n. 1, p. 120-128, 2004.

STATISTIC FOR WINDOWS. Copyright $\odot$ Sta Soft. Inc., Tulsa. Versão 5.0. 1995.

STONE, H.; SIDEL, J. L. Sensory avaluation practices. Flórida: Academic Press, Inc., 1985. 311p.

WASZCZYNSKYJ, K.; NELSEN, T. Collaborative study on sensory and volatile compound analysis of oils. Journal of the American Oil Chemists Society, v. 73, n. 2, p. 157-166, feb. 1996. 